\title{
Lagrangian Dynamics of Spinning Particles and Polarized Media in General Relativity*
}

\author{
Ian Bailey \\ Mathematics Department, University of Alberta, Edmonton, Canada
}

Werner Israel

Theoretical Physics Institute, Physics Department, University of Alberta, Edmonton, Canada

Received March 26, 1974; in revised form November 12, 1974

\begin{abstract}
The general form of the Lagrangian equations of motion is derived for a spinning particle having arbitrary multipole structure in arbitrary external fields. It is then shown how these equations, together with the complete system of field equations can be recovered from a fourdimensional action integral representing a polarized dustlike medium interacting with an arbitrary set of fields. These general results are then specialized to the case of Einstein-Maxwell fields in order to obtain the general-relativistic extension of Lorentz's dielectric theory.
\end{abstract}

\section{Introduction}

This paper attempts to answer and to trace the connections between two principal questions:

What are the equations of motion of a spinning multipole test particle in given external fields?

For a continuous medium with internal spin and multipole structure, what are the phenomenological field equations relating the fields generated by the medium to its statistical bulk properties? (The classical prototype is Lorentz's dielectric theory.)

Both questions have long histories of research behind them. The close link between them has been stressed in a recent note by one of us [1]: for a gaseous medium in a self-consistent Einstein-Maxwell background field, the field equations are delimited to a virtually unique form by the requirement that they be compatible with conservation laws derived from the equations of motion of the constituent particles. In the present, more complete and more general discussion, we show how the general form of both the equations of motion and the field equations can be derived from a unified Lagrangian viewpoint.

Equations of motion for spinning test bodies have traditionally been derived by two principal methods ${ }^{1}$ :

(i) Multipole Formalism for Extended Body or Equivalent Schwartz Distribution. This method, due originally to Mathisson [5] and developed further by Papapetrou [6], Taub [7], Dixon [8], Madore [9], Suttorp and de Groot [2], and others $[10]$, proceeds essentially by integrating the conservation identities

* Work partially supported by the National Research Council of Canada.

1 For reviews and extensive bibliographies (especially of the special-relativistic literature), see the books by Suttorp and de Groot [2], Halbwachs [3], and Corben [4]. 
for the total energy tensor $T^{\alpha \beta}$ over space-like sections $\Sigma$ of the particle's worldtube. In any such formalism the definitions of gross particle properties necessarily involve a number of arbitrary elements. One has to settle on conventions for (a) allocation of the interaction energy when splitting $T^{\alpha \beta}$ into an "internal" matter part $T_{\text {(mat) }}^{\alpha \beta}$ - either canonical, or symmetrized to include internal spin contributions - and an "external" field part; (b) choice of tube sections $\Sigma$ in the specialrelativistic integral for the particle's 4-momentum,

$$
p^{\alpha}=-\int_{\Sigma} T_{(\text {mat })}^{\alpha \beta} n_{\beta} d \Sigma
$$

or its general-relativistic equivalent; (c) choice of a central world-line for defining the particle's 4-velocity $u^{\alpha}$ and spin angular momentum $S^{\lambda \mu}$. (Different choices (c) lead to different so-called "auxiliary conditions", such as $S^{\lambda \mu} u_{\mu}=0, S^{\lambda \mu} p_{\mu}=0$ and others $[6,8 \mathrm{a}, 2,11]$. Because of the obvious physical difficulty of localizing the particle to within a distance $\sim(\operatorname{spin}) / c \times$ (mass), which exists at both the macroscopic and elementary-particle levels, this uncertainty is unlikely to be fully resolved except by formal convention.) The form of the equations of motion naturally depends to some extent on the definitions of the quantities entering them, and a fair variety of seemingly different formalisms have been obtained in this way.

(ii) Equations of Motion from an Action Principle. Frenkel [12], with choice of a special Lagrangian, and more generally Barut [13], without specifying the functional form of the Lagrangian, have derived special-relativistic equations of motion for spinning particles in electromagnetic fields; both assume $S^{\lambda \mu} u_{\mu}=0$. In general relativity, Künzle [14] and Souriau [15] have recently obtained equations for spinning dipoles in Einstein-Maxwell fields from a Lorentz-invariant pre-symplectic 1 -form; they adopt the auxiliary condition $S^{\lambda \mu} p_{\mu}=0$.

Yet other derivations of particle equations of motion proceed on a more-orless ad hoc basis [16].

In the case of continuous media, the dynamics of interaction with the electromagnetic field has been an active and controversial area since the earliest days of special relativity ${ }^{2}$. Rival and apparently conflicting proposals put forward by Minkowski, Abraham, and Einstein and Laub [20] for the localization of electromagnetic energy and momentum in a dielectric medium were a subject of debate for many years $[17,2]$. Not until the 1960 's was it clearly recognized $[18,19,21,22]$ that the question cannot be meaningfully handled without detailed consideration of the material terms. Only the total (symmetric, conserved) energy-momentum tensor has a fundamental significance by virtue of its coupling with the gravitational field. Its split, for the purpose of phenomenological description, into "matter" and "field" parts involves arbitrary, conventional elements relating to the allocation of the interaction terms. Of course, phenomenological descriptions retain their usefulness; in particular, the statistical treatment of a medium with internal spin interacting with the gravitational field, and having a corpuscular structure at the macroscopic level (primeval galaxies, black holes, turbulent eddies) is of potential interest for application to the early cosmological "soup", but has not as yet received much attention [1].

2 For critical reviews and extensive references see the books by Suttorp and de Groot [2], Pauli [17], Møller [18], and Penfield and Haus [19]. 
The first goal of the present work is to derive the general form of the Lagrangian equations of motion for a spinning particle having arbitrary multipole structure in arbitrary external fields [Eqs. (22) and (23) of Section III]. Our treatment is independent of special interaction models in that we do not specify the functional form of the Lagrangian, but only the variables on which the Lagrangian depends. This generality of the Lagrangian also makes a priori commitment to a particular auxiliary condition unnecessary. The resulting equations represent the canonical substructure to which every detailed model of particle motion must conform.

In Section IV we extend this analysis to the field dynamics of continua. We consider a four-dimensional action integral in which the Lagrangian density is an unspecified function of a vector field $n u^{\alpha}$ (representing a particle flux), an orthonormal tetrad field $e_{\alpha}^{(a)}$ (representing a set of spin axes) and arbitrary field variables. Physically, this represents a dustlike medium interacting with the external fields treated as self-consistent backgrounds. Variation with respect to the sixteen tetrad components $e_{x}^{(a)}$ yields six equations for the spin angular momentum and the ten gravitational field equations. The four equations of translational motion are then obtainable (modulo the nongravitational field equations) either from the contracted Bianchi identities or by "variation of the world-lines" (Section IV, Part 3). We thus recover the equations of motion previously derived in Section III together with the complete system of equations relating the fields to their sources.

In Section $\mathrm{V}$ these general considerations are applied specifically to a medium interacting with an Einstein-Maxwell field. The results give a clear formal expression to the general relativistic extension of Lorentz dielectric theory. Interestingly, a complete analogy emerges between the appearance of the usual polarization terms in the phenomenological Maxwell-Lorentz equations and the appearance in the gravitational equations of the well-known Belinfante-Rosenfeld symmetrization terms [23] involving the spin flux. It is thus proper to interpret these terms as a "gravitational polarization tensor" [1].

While it would have been straightforward to generalize our considerations to fluid and elastic media ${ }^{3}$, we have resisted the temptation to do so in this paper in order to exhibit as clearly as possible the interrelationship between the field equations for dustlike media and the equations of motion for free particles.

Also left out of consideration here are the "torsion theories" of Weyl [26], Sciama [27], and Kibble [28], recently reviewed and developed by Hehl [29] and Trautman [30], which attempt to relate the spin of elementary particles to the skew part of a metric-preserving, non-Riemannian affine connexion. Such theories can be reformulated as Riemannian theories with an auxiliary torsion tensor, which then appears (quadratically) as a complicated supplementary term in the energy-momentum tensor on the right-hand side of the conventional Einstein field equations. Since gravitational effects of elementary particle spins are completely insignificant ${ }^{4}$ below densities of order $10^{55} \mathrm{gm} / \mathrm{cm}^{3}$ [32], both the torsion theories and the simpler conventional theories $[23,1]$ effectively imply validity of the usual (spin-free) form of the energy-momentum tensor at the ordinary macroscopic level, and lead to the same phenomenological polarization theory for aggregates of "particles" of astronomical size.

\footnotetext{
3 See Refs. [24], [25], and [1] for some partial extensions along these lines.

4 But see [31] for a different point of view.
} 


\section{Definitions and General Identities}

We begin by collecting a number of general formulas for sets of relative tensor fields $\Phi_{A}\left(x^{\mu}\right)$, and then develop the identities (cf. Belinfante and Rosenfeld [23]) which flow from the condition that a function $L\left(\Phi_{A}\right)$ be a (relative) scalar.

Greek indices run from 1 to 4 . Capitalized Latin indices $A, B, \ldots$ do double duty as labels $\left(A_{L}, B_{L}, \ldots\right)$ and as generic representations of sets of tensor component indices. Thus,

$$
\Phi_{A} \equiv\left(\Phi_{A_{L}}\right)_{\alpha_{1} \ldots \alpha_{m}}^{\alpha_{m+1} \ldots \alpha_{n}} ; \quad \Phi^{A} \equiv\left(\Phi_{A_{L}}\right)^{\alpha_{1} \ldots \alpha_{m}}{ }_{\alpha_{m+1} \ldots \alpha_{n}}
$$

where $m, n$ depend on the label $A_{L}$. Sometimes, as in (13) below, a generic index $A$ is printed bold face as a means of indicating that $\boldsymbol{A}$ and $A$ have different labelranges. A repeated generic index implies summation over the respective tensor indices and over the label-range concerned.

Under the co-ordinate transformation $\bar{x}^{\lambda}=\bar{x}^{\lambda}\left(x^{\mu}\right)$, the relative tensor $\Phi_{A}$ transforms linearly:

$$
\bar{\Phi}_{A}(\bar{x})=\Lambda_{A}{ }^{B}\left(X^{\varrho}{ }_{\sigma}\right) \Phi_{B}(x), \quad X_{\sigma}^{\varrho} \equiv \partial \bar{x}^{\varrho} / \partial x^{\sigma} .
$$

[Of course, $A_{A}{ }^{B}$ vanishes unless $A_{L}=B_{L}$ so the summation over $B$ in (1) does not couple different tensors.] The infinitesimal generators of the transformations (1) are

$$
\left(I_{A}{ }^{B}\right)_{\varrho}{ }^{\sigma} \equiv\left(\partial \Lambda_{A}{ }^{B} / \partial X^{\varrho}{ }_{\sigma}\right)_{X} \varrho_{\sigma}=\delta_{\sigma}{ }^{\varrho} .
$$

Explicit construction of these generators follows easily from the recursion formulas $(I)_{\varrho}{ }^{\sigma}=-w \delta_{\varrho}^{\sigma}$ for a relative scalar $\phi$ of weight

$$
\begin{gathered}
\left.w \quad \text { (i.e., } \bar{\phi}(\bar{x})=\phi(x)|\partial x / \partial \bar{x}|^{w}\right) ; \\
\left(I_{A \alpha}{ }^{B \beta}\right)_{\varrho}{ }^{\sigma}=\left(I_{A}{ }^{B}\right)_{\varrho}{ }^{\sigma} \delta_{\alpha}^{\beta}-\delta_{A}^{B} \delta_{\alpha}^{\sigma} \delta_{\varrho}^{\beta} ; \\
\left(I_{A \beta}^{\alpha B}\right)_{\varrho}{ }^{\sigma}=\left(I_{A}{ }^{B}\right)_{\varrho}{ }^{\sigma} \delta_{\beta}^{\alpha}+\delta_{A}^{B} \delta_{\varrho}^{\alpha} \delta_{\beta}^{\sigma},
\end{gathered}
$$

where $\delta_{A}^{B}=1$ if $A_{L}=B_{L}$ and the respective tensor indices are equal, and $\delta_{A}^{B}=0$ otherwise. The group property $\Lambda_{A}{ }^{B}\left(Y^{\lambda}{ }_{\mu} X^{\mu}{ }_{v}\right)=\Lambda_{A}{ }^{C}(Y) \Lambda_{C}{ }^{B}(X)$ yields

$$
\left(I_{A}{ }^{C}\right)_{\lambda}{ }^{\mu}\left(I_{C}{ }^{B}\right)_{\alpha}{ }^{\beta}-\left(I_{A}{ }^{C}\right)_{\alpha}{ }^{\beta}\left(I_{C}{ }^{B}\right)_{\lambda}{ }^{\mu}=\left(I_{A}{ }^{B}\right)_{\lambda}{ }^{\beta} \delta_{\alpha}^{\mu}-\left(I_{A}{ }^{B}\right)_{\alpha}{ }^{\mu} \delta_{\lambda}^{\beta} \text {. }
$$

Useful formulas which will be required in the sequel are

$$
\begin{aligned}
\Phi_{A \mid \tau} & =\partial_{\tau} \Phi_{A}+\Gamma_{\sigma \tau}^{\varrho}\left(I_{A}{ }^{B}\right)_{\varrho}{ }^{\sigma} \Phi_{B}, \\
\Phi_{A \mid \mu \nu}-\Phi_{A \mid v \mu} & =-R^{\varrho}{ }_{\sigma \mu \nu}\left(I_{A}{ }^{B}\right)_{\varrho}{ }^{\sigma} \Phi_{B},
\end{aligned}
$$

where the stroke denotes covariant differentiation with respect to a symmetric affine connexion. From (1) and (2) the change of $\Phi_{A}$ under the infinitesimal coordinate transformation $\bar{x}^{\varrho}=x^{\varrho}+\xi^{\varrho}(x)$ is

$$
\bar{\Phi}_{A}(\bar{x})-\Phi_{A}(x)=\left(I_{A}{ }^{B}\right)_{\varrho}{ }^{\sigma} \Phi_{B}\left(\partial_{\sigma} \xi^{\varrho}\right) .
$$

From (8) we derive at once

$$
\frac{\partial \boldsymbol{L}}{\partial \Psi_{\boldsymbol{A}}}\left(I_{\boldsymbol{A}}^{\boldsymbol{B}}\right)_{\varrho}{ }^{\sigma} \Psi_{\boldsymbol{B}}+w \delta_{\varrho}^{\sigma} \boldsymbol{L}=0
$$


as the condition that the function $L\left(\Psi_{\boldsymbol{A}}\right)$ of the relative tensors $\Psi_{\boldsymbol{A}}$ transforms as a relative scalar of weight $w$.

A more restrictive identity can be derived from (9) in the case of a scalar density $(w=1) \boldsymbol{L}\left(\psi_{A}, \psi_{A \mid \varrho}\right)$ depending on a set of relative tensor fields $\psi_{A}$ and their first ${ }^{5}$ covariant derivatives. We define the variational derivative

where

$$
\delta \boldsymbol{L} / \delta \psi_{A} \equiv \boldsymbol{L}^{A}-\boldsymbol{L}_{\mid \alpha}^{A \alpha},
$$

$$
\boldsymbol{L}^{A} \equiv \partial \boldsymbol{L} / \partial \psi_{A}, \quad \boldsymbol{L}^{A \alpha} \equiv \partial \boldsymbol{L} / \partial \psi_{A \mid \alpha},
$$

and the tensor densities

$$
\boldsymbol{t}_{\varrho}{ }^{\sigma} \equiv \boldsymbol{L} \delta_{\varrho}^{\sigma}-\psi_{A \mid \varrho} \boldsymbol{L}^{A \sigma} ; \quad \boldsymbol{U}^{\tau \sigma}{ }_{\varrho} \equiv \boldsymbol{L}^{A \tau}\left(I_{A}{ }^{B}\right)_{\varrho}{ }^{\sigma} \psi_{B} .
$$

Further, we note from ( 4 a) that

$$
\boldsymbol{L}^{A \alpha}\left(I_{A \alpha}{ }^{B \beta}\right)_{\varrho}{ }^{\sigma} \psi_{B \mid \beta}=\boldsymbol{L}^{A \alpha}\left(I_{A}{ }^{B}\right)_{\varrho}{ }^{\sigma} \psi_{B \mid \alpha}-\boldsymbol{L}^{A \sigma} \psi_{A \mid \varrho} .
$$

Then (9), with

$$
\Psi_{\boldsymbol{A}} \equiv\left(\psi_{A}, \psi_{\boldsymbol{A} \mid \alpha}\right)
$$

yields at once the identity

$$
\boldsymbol{U}_{\varrho \mid \tau}^{\tau \sigma}+\boldsymbol{t}_{\varrho}{ }^{\sigma}+\frac{\delta \boldsymbol{L}}{\delta \psi_{A}}\left(I_{A}{ }^{B}\right)_{\varrho}{ }^{\sigma} \psi_{B}=0
$$

valid for an arbitrary scalar density $\boldsymbol{L}\left(\psi_{A}, \psi_{A \mid \alpha}\right)$.

\section{Spinning Multipole in Given External Fields}

In this section, we derive the general form of the Lagrangian equations of motion for a spinning test particle with arbitrary multipole structure in a given Riemannian space-time and under the influence of a given set of external fields $\phi_{A}$.

Let $x^{\mu}=x^{\mu}(t)$ be the equation of the particle world-line in terms of an arbitrary scalar parameter $t, \tau$ the proper time, and

$$
u^{\mu}=d x^{\mu} / d \tau, \quad v^{\mu}=d x^{\mu} / d t
$$

the normalized and unnormalized 4-velocities respectively. The spin of the particle is described by the gyration of an orthonormal tetrad $e^{(a)}(t)$ defined on the world-line:

$$
\eta_{a b} e_{\alpha}^{(a)} e_{\beta}^{(b)}=g_{\alpha \beta}, \quad \eta_{a b}=\operatorname{diag}(1,1,1,-1) \quad(a, b, \ldots=1 \quad \text { to } 4) .
$$

The equations of motion are assumed to be derivable from a parameterinvariant action principle $\delta \int L d t=0$, for variations of $x^{\mu}(t)$ and $e_{\alpha}^{(a)}(t)$ with fixed endpoints $x^{\mu}\left(t_{i}\right), e_{\mu}^{(a)}\left(t_{i}\right)(i=1,2)$. The Lagrangian is an unspecified scalar function

$$
L=L\left(v^{\mu}, e_{\alpha}^{(a)}, \dot{e}_{\alpha}^{(a)}, \Phi_{\boldsymbol{A}}\right),
$$

5 The case where higher-order derivatives occur is not needed immediately and its consideration is deferred to Section VI. 
where $\dot{e}_{\alpha}^{(a)} \equiv \delta e_{\alpha}^{(a)} / \delta t$ (absolute derivative) and the set of tensors $\Phi_{\boldsymbol{A}}$ comprises the external fields $\phi_{A}$, the Riemann tensor $R_{\beta \gamma \delta}^{\alpha}$ and their symmetrized ${ }^{6}$ covariant derivatives:

$$
\Phi_{A}=\left(\phi_{A}, \phi_{A \mid \alpha}, \ldots \phi_{A \mid\left(\alpha_{1} \ldots \alpha_{n}\right)}, \ldots ; R_{\beta \gamma \delta}^{\alpha}, \ldots, R_{\beta \gamma \delta \mid\left(\varepsilon_{1} \ldots \varepsilon_{n}\right)}^{\alpha} \ldots\right) .
$$

We have assumed that explicit dependence of $L$ on $g_{\alpha \beta}$ has been eliminated by the use of (15).

We define the canonical momentum $P_{\mu}$, the spin angular momentum $S^{\varrho \sigma}$ $=S^{[\varrho \sigma]}$ and the multipole moments $M^{\boldsymbol{A}}$ by

$$
P_{\mu}=\frac{\partial L}{\partial v^{\mu}}, \quad S_{\varrho}^{\sigma}=2 e_{[\varrho}^{(a)} \frac{\partial L}{\partial \dot{e}_{\sigma]}^{(a)}}, \quad M^{\boldsymbol{A}}=\frac{\partial L}{\partial \Phi_{\boldsymbol{A}}} \quad(t=\tau) .
$$

Since the components $\Phi_{\boldsymbol{A}}$ are usually constrained by algebraic symmetries, a word about the definition of the partial derivatives $M^{\boldsymbol{A}}=\partial L / \partial \Phi_{\boldsymbol{A}}$ is in order. These are in all cases defined so that $d L=M^{\boldsymbol{A}} d \Phi_{\boldsymbol{A}}$. If the $\Phi_{\boldsymbol{A}}$ possess algebraic symmetries, we consider only variations $d \Phi_{\boldsymbol{A}}$ which respect these symmetries. The resulting arbitrariness in the coefficients $M^{A}$ is then resolved by requiring that $M^{\boldsymbol{A}}$ share the symmetries of $\Phi_{\boldsymbol{A}}$. These conventions lead to formulas like $\partial F_{12} / \partial F_{12}=\frac{1}{2}=-\partial F_{12} / \partial F_{21}$ for a skew symmetric tensor $F_{\alpha \beta}$. It follows also that the $2^{n+2}$-pole gravitational moment tensor ${ }^{7}$

$$
Q_{\alpha}^{\beta \gamma \delta \varepsilon_{1} \ldots \varepsilon_{n}}=\partial L / \partial R_{\beta \gamma \delta \mid\left(\varepsilon_{1} \ldots \varepsilon_{n}\right)}^{\alpha}
$$

is symmetric in its last $n$ indices, and has the algebraic symmetries of the Riemann tensor in its first four indices.

The equations of motion for the spin $S^{\varrho \sigma}$ are obtained by variation of $e_{\alpha}^{(a)}(t)$ with fixed end-values, holding the world-line fixed. Since in this section (only) we treat the space-time geometry as prescribed, Eq. (15) impose 10 constraints on the 16 variations $\delta e_{\alpha}^{(a)}$. The resulting equations of motion reduce, after eliminating the Lagrange multipliers, to the 6 equations

$$
\frac{\partial L}{\partial e_{[\sigma}^{(a)}} e_{\varrho]}^{(a)}=0, \quad \frac{\partial L}{\partial e_{\sigma}^{(a)}} \equiv \frac{\partial L}{\partial e_{\sigma}^{(a)}}-\frac{\delta}{\delta t}\left(\frac{\partial L}{\partial \dot{e}_{\sigma}^{(a)}}\right) .
$$

On the other hand, the identity (9) with $w=0$ gives

$$
\frac{\partial L}{\partial v^{\varrho}} v^{\sigma}-\frac{\partial L}{\partial e_{\sigma}^{(a)}} e_{\varrho}^{(a)}-\frac{\partial L}{\partial \dot{e}_{\sigma}^{(a)}} \dot{e}_{\varrho}^{(a)}+\frac{\partial L}{\partial \Phi_{\boldsymbol{A}}}\left(I^{\boldsymbol{A}}{ }_{\boldsymbol{B}}\right)_{\varrho}{ }^{\sigma} \Phi_{\boldsymbol{B}}=0 .
$$

Differentiating the second of (18), we find with the aid of (21) (with $t=\tau$ ) that Eq. (20) is equivalent to

$$
\frac{1}{2} \delta S^{\varrho \sigma} / \delta \tau=P^{[\varrho} \mathcal{U}^{\sigma]}+M^{\boldsymbol{A}}\left(I_{\boldsymbol{A}}^{\boldsymbol{B}}\right)^{[\varrho \sigma]} \Phi_{\boldsymbol{B}} .
$$

${ }^{6}$ This is not a restriction, since an unsymmetrized covariant derivative can always be reduced to a set of the form (17) with the aid of the Ricci commutation relations (7).

7 This corresponds (apart from a numerical factor) to the "reduced" moment integral $J^{\varepsilon_{1} \ldots \varepsilon_{n} \alpha \beta ; \delta}$ defined for extended mass distributions by Dixon (Ref. [8b], Eq. (5.28)). 
The equations of motion for the linear momentum are obtained from an infinitesimal displacement of the world-line, holding $e_{\alpha}^{(a)}$ fixed by parallel propagation. We consider a 1-parameter family of time-like curves $x^{\mu}(t, \varepsilon)$ with orthonormal tetrads $e_{\alpha}^{(a)}(t, \varepsilon)$ defined on them, and extremize $I(\varepsilon)=\int_{t_{1}}^{t_{2}} L d t$ subject to fixed $x^{\mu}\left(t_{i}, \varepsilon\right)=x^{\mu}\left(t_{i}, 0\right) \quad(i=1,2), \delta e_{\alpha}^{(a)} / \delta \varepsilon=0$. This yields, for arbitrary variations $\partial x^{\mu} / \partial \varepsilon$,

$$
\int_{t_{1}}^{t_{2}}\left(\frac{\partial L}{\partial v^{\mu}} \frac{\delta v^{\mu}}{\delta \varepsilon}+\frac{\partial L}{\partial \dot{e}_{\sigma}^{(a)}} \frac{\delta}{\delta \varepsilon}\left(\frac{\delta e_{\sigma}^{(a)}}{\delta t}\right)+\frac{\partial L}{\partial \Phi_{\boldsymbol{A}}} \Phi_{\boldsymbol{A} \mid \mu} \frac{\partial x^{\mu}}{\partial \varepsilon}\right) d t=0
$$

Noting

$$
\frac{\delta v^{\mu}}{\delta \varepsilon}=\frac{\delta}{\delta t}\left(\frac{\partial x^{\mu}}{\partial \varepsilon}\right), \quad \frac{\delta}{\delta \varepsilon}\left(\frac{\delta e_{\sigma}^{(a)}}{\delta t}\right)=e_{\varrho}^{(a)} R_{\sigma \lambda \mu}^{\varrho} v^{\lambda} \frac{\partial x^{\mu}}{\partial \varepsilon},
$$

and integrating by parts gives the equations of motion

$$
\delta P_{\mu} / \delta \tau=\frac{1}{2} R_{\varrho \sigma \lambda \mu} S^{\varrho \sigma} u^{\lambda}+M^{A} \Phi_{\boldsymbol{A} \mid \mu},
$$

where we have set $t=\tau$ on the extremizing curve.

To facilitate comparison with the results of the next section, we note in conclusion that the relation

$$
\delta e_{\alpha}^{(a)} / \delta t=\left(\delta e_{\alpha}^{(a)} / \delta \tau\right)\left(-\eta_{a b} e_{\alpha}^{(a)} e_{\beta}^{(b)} v^{\alpha} v^{\beta}\right)^{\frac{1}{2}}
$$

can be used to re-express $L$ as a new function

$$
L=L_{1}\left(v^{\mu}, e_{\alpha}^{(a)}, \dot{e}_{\alpha}^{(a)}(\tau), \Phi_{\boldsymbol{A}}\right) ; \quad \dot{e}_{\alpha}^{(a)}(\tau) \equiv \delta e_{\alpha}^{(a)} / \delta \tau
$$

which is homogeneous of first degree in its first argument $v^{\mu}$ (because of the parameter-invariance of the action integral). The partial derivatives (18) now appear as

$$
\begin{aligned}
S_{\varrho}{ }^{\sigma} & =2 e_{[\varrho}^{(a)} \frac{\partial L_{1}}{\partial \dot{e}_{\sigma]}^{(a)}(\tau)}, M^{\boldsymbol{A}}=\frac{\partial L_{1}}{\partial \Phi_{\boldsymbol{A}}} \\
P_{\mu} & =\frac{\partial L_{1}}{\partial v^{\mu}}+\frac{\partial L_{1}}{\partial \dot{e}_{\alpha}^{(a)}(\tau)} \dot{e}_{\alpha}^{(a)} u_{\mu} \quad(t=\tau) .
\end{aligned}
$$

\section{Field Dynamics of Continua: First-derivative Coupling}

The objective of this section is a unified derivation of the equations of motion (22), (23) together with a system of field equations for the applied fields from a four-dimensional action integral.

The Lagrangian density (precise functional form not specified) represents a set of free fields $g_{\alpha \beta}, \phi_{A}$ interacting with a continuous medium having a simple structure. The medium may be visualized as consisting, at the "atomic" level, of coherently moving, spinning particles which interact only through the external fields, treated as self-consistent backgrounds. Its history is thus completely described by a "numerical flux"

$$
N^{\alpha}(x)=\sqrt{-g} n u^{\alpha}, \quad u_{\alpha} u^{\alpha}=-1
$$


and an orthonormal tetrad field $e_{\alpha}^{(a)}(x)$. (Our treatment is easily generalized; for example, a medium with internal stresses produced by incoherently moving particles can be handled by a sum $\Sigma_{r}$ of action integrals containing fluxes $\boldsymbol{N}^{\alpha}(x, r)$ and tetrads $e_{\alpha}^{(a)}(x, r)$, where $r$ is a discrete or continuous index. This leads to equations of motion of the form (22), (23) for each "stream" $r$ and to the same field equations. Compare Ref. [1].)

We accordingly postulate that the field equations and equations of motion are obtainable from the action integral

$$
I=\int \boldsymbol{L}\left(\psi_{\boldsymbol{A}}, \psi_{\boldsymbol{A} \mid \alpha}\right) d^{4} x,
$$

in which the scalar density $\boldsymbol{L}$ depends on the fields

$$
\psi_{\boldsymbol{A}}=\left\{\boldsymbol{N}^{\alpha}, e_{\alpha}^{(a)}, \phi_{A}, R_{\beta \gamma \delta}^{\alpha}\right\}
$$

and on $e_{\alpha \mid \beta}^{(a)}$. In general, $L$ will depend also on covariant derivatives of $\phi_{A}$ and $R^{\alpha}{ }_{\beta \gamma \delta}$. We shall simplify the discussion in this section by assuming that this dependence is restricted to first derivatives $\phi_{A \mid \alpha}$ and that no derivatives of $R_{\beta \gamma \delta}^{\alpha}$ enter. This is still sufficiently general to cover most cases of practical interest. (For the analysis of the general case, see Section VI.)

In this formulation the sixteen-component tetrad field $e_{\alpha}^{(a)}$ plays a dual role: the ten symmetrized products $\eta_{a b} e_{\alpha}^{(a)} e_{\beta}^{(b)} \equiv g_{\alpha \beta}$ define the metric and determine the gravitational field, while the six angular velocities $\omega^{a b}=e^{(a) \alpha} e_{\alpha \mid \beta}^{(b)} u^{\beta}$ determine the internal spin of the medium.

\section{Spin Equations and Gravitational Field Equations Derived from Variation of Tetrad}

Under an arbitrary variation $\delta e_{\sigma}^{(a)}$ of the tetrad field, and the accompanying variation

$$
\delta g_{\varrho \sigma}=2 \eta_{a b} e_{(\varrho}^{(b)} \delta e_{\sigma)}^{(a)}
$$

of the metric, the variation $\delta_{(e)} \boldsymbol{L}$ gets contributions from three sources: (i) explicit dependence of $\boldsymbol{L}$ on $e_{\alpha}^{(a)}, e_{\alpha \mid \beta}^{(a)}$; (ii) variation of the affine connexion hidden in the covariant derivatives $e_{\alpha \mid \beta}^{(a)}, \phi_{A \mid \alpha}$; and (iii) variation of $R_{\beta \gamma \delta}^{\alpha}$.

Accordingly, we have

$$
\delta_{(e)} \boldsymbol{L}=\left(\delta \boldsymbol{L} / \delta e_{\sigma}^{(a)}\right) \delta e_{\sigma}^{(a)}+\boldsymbol{U}_{\varrho}^{\tau \sigma} \delta \Gamma_{\sigma \tau}^{\varrho}+\boldsymbol{Q}_{\alpha}^{\beta \gamma \delta} \delta R_{\beta \gamma \delta}^{\alpha}+(\mathrm{div}),
$$

where $\delta \boldsymbol{L} / \delta \psi_{\boldsymbol{A}}$ and $\boldsymbol{U}^{\tau \sigma}{ }_{\varrho}$ were defined in (10) and (12),

$$
\boldsymbol{Q}_{\alpha}^{\beta \gamma \delta}=\partial \boldsymbol{L} / \partial \boldsymbol{R}_{\beta \gamma \delta}^{\alpha},
$$

and (div) represents a divergence $\partial_{\alpha}(\ldots)$.

To re-express the last two terms of (30) in terms of $\delta e_{\sigma}^{(a)}$, we note that

$$
\delta R_{\beta \gamma \delta}^{\alpha}=2\left(\delta \Gamma_{\beta[\delta \mid \gamma]}^{\alpha}\right)_{\mid \gamma]}, \quad \delta \Gamma_{\sigma \tau}^{\varrho}=(\delta g)_{(\sigma \mid \tau)}^{\varrho}-\frac{1}{2}(\delta g)_{\sigma \tau}{ }^{l} \varrho
$$

enable us to write the identities

$$
\begin{gathered}
\boldsymbol{Q}_{\alpha}^{\beta \gamma \delta} \delta R_{\beta \gamma \delta}^{\alpha}=-2 \boldsymbol{Q}_{\varrho}{ }^{\sigma[\mu \tau]}{ }_{\mid \mu} \delta \Gamma_{\sigma \tau}^{\varrho}+(\mathrm{div}) \\
\boldsymbol{U}^{\tau \sigma}{ }_{\varrho} \delta \Gamma_{\sigma \tau}^{\varrho}=\frac{1}{2}\left\{\frac{1}{2}\left(\boldsymbol{S}^{\sigma \tau \varrho}+\boldsymbol{S}^{\varrho \tau \sigma}\right)-\boldsymbol{U}^{\tau(\varrho \sigma)}\right\}_{\mid \tau} \delta g_{\varrho \sigma}+(\mathrm{div}),
\end{gathered}
$$


valid for arbitrary tensor densities $\boldsymbol{Q}_{\alpha}{ }^{\beta \gamma \delta}, \boldsymbol{U}^{\tau \sigma}{ }_{\varrho}$. We have defined the "spin flux"

$$
S^{\sigma \tau \varrho}=2 U^{\varrho[\sigma \tau]} .
$$

The action principle $\delta_{(e)} I=0$ now gives, with the aid of (30), (32), (33), and (29),

$$
\left(\delta \boldsymbol{L} / \delta e_{\sigma}^{(a)}\right) e^{(a) \varrho}=\left\{\boldsymbol{U}^{\tau(\varrho \sigma)}-\frac{1}{2}\left(\boldsymbol{S}^{\sigma \tau \varrho}+\boldsymbol{S}^{\varrho \tau \sigma}\right)\right\}_{\mid \tau}-4 \boldsymbol{Q}_{\mid \mu \lambda}^{\lambda(\varrho \sigma) \mu} .
$$

The symmetry of the right-hand side in $\varrho, \sigma$ gives at once the six equations of motion for spin:

$$
\left(\delta \boldsymbol{L} / \delta e_{[\sigma}^{(a)}\right) e_{\varrho]}^{(a)}=0 .
$$

The gravitational field equations comprise the essential content of the remaining ten equations of (35). As a first step towards reducing these to a familiar form, we call on the identity (14), which in the present context reads

$$
\frac{\delta \boldsymbol{L}}{\delta e_{\sigma}^{(a)}} e_{\varrho}^{(a)}=\frac{\partial \boldsymbol{L}}{\partial \boldsymbol{N}^{\varrho}} \boldsymbol{N}^{\sigma}-\frac{\partial \boldsymbol{L}}{\partial \boldsymbol{N}^{\alpha}} \boldsymbol{N}^{\alpha} \delta_{\varrho}^{\sigma}+\boldsymbol{U}^{\tau \sigma}{ }_{\varrho \mid \tau}+\boldsymbol{t}_{\varrho}{ }^{\sigma}+\boldsymbol{Q}^{A}\left(I_{A}{ }^{B}\right)_{\varrho}{ }^{\sigma} R_{B}
$$

modulo the nongravitational field equations

$$
\delta \boldsymbol{L} / \delta \phi_{A}=0
$$

The last term of (37) has the explicit form

$$
Q^{A}\left(I_{A}{ }^{B}\right)_{\varrho}{ }^{\sigma} R_{B}=Q_{\varrho}^{\beta \gamma \delta} R_{\beta \gamma \delta}^{\sigma}-3 Q_{\alpha}{ }^{\sigma \gamma \delta} R_{\varrho \gamma \delta}^{\alpha} .
$$

Elimination of the common left-hand sides of (35) and (37) yields

$$
\begin{aligned}
\left(\partial \boldsymbol{L} / \partial \boldsymbol{N}^{\alpha}\right) \boldsymbol{N}^{\alpha} \delta_{\varrho}^{\sigma}-\boldsymbol{t}_{\varrho}{ }^{\sigma}-Q^{A}\left(I_{A}{ }^{B}\right)_{\varrho}{ }^{\sigma} R_{B}=\left(\partial \boldsymbol{L} / \partial \boldsymbol{N}^{\varrho}\right) \boldsymbol{N}^{\sigma} \\
+\frac{1}{2}\left(\boldsymbol{S}^{\sigma \tau}{ }_{\varrho}+\boldsymbol{S}_{\varrho}{ }^{\tau \sigma}+\boldsymbol{S}^{\sigma}{ }_{\varrho}{ }^{\tau}\right)_{\mid \tau}+4 Q^{\lambda(\sigma)^{\mu}{ }_{\mid \mu \lambda} .}
\end{aligned}
$$

\section{Matter and Field Decomposition of Lagrangian}

To proceed further with the reduction of Eq. (40), we decompose $\boldsymbol{L}$ into a sum

$$
\begin{gathered}
\boldsymbol{L}=\boldsymbol{L}_{1}\left(\boldsymbol{N}^{\mu}, e_{\alpha}^{(a)}, \dot{e}_{\alpha}^{(a)}, \Phi_{A}\right)+\boldsymbol{L}_{2}\left(\phi_{A}, \phi_{A \mid \alpha}, g_{\alpha \beta}\right)+\boldsymbol{L}_{3}, \\
\dot{e}_{\alpha}^{(a)} \equiv e_{\alpha \mid \mu}^{(a)} u^{\mu}, \quad \Phi_{\boldsymbol{A}} \equiv\left(\phi_{A}, \phi_{A \mid \alpha}, R_{\beta \gamma \delta}^{\alpha}\right),
\end{gathered}
$$

in which $L_{1}$ represents the matter and its interaction with the applied fields, and $L_{2}, L_{3}$ are the free-field Lagrangians for $\phi_{A}$ and the gravitational field respectively. We now assume:

(i) That $\boldsymbol{L}_{1}\left(\boldsymbol{N}^{\mu}, \ldots\right) \equiv L_{1}\left(\boldsymbol{N}^{\mu}, \ldots\right)$ is homogeneous of first degree in its first argument, so that

$$
\boldsymbol{L}_{1}=n \sqrt{-g} L_{1}\left(u^{\mu}, e_{\alpha}^{(a)}, \dot{e}_{\alpha}^{(a)}, \Phi_{\boldsymbol{A}}\right) .
$$

This amounts to assuming that the flux of material 4-momentum and spin is proportional to the particle density $n$, and is clearly satisfied for dustlike material (no direct inter-particle interactions).

(ii) That the gravitational Lagrangian is essentially the curvature scalar:

$$
16 \pi \boldsymbol{L}_{3}=-\sqrt{-g} R \text {. }
$$


According to Lovelock's theorem [33], any choice $\boldsymbol{L}_{3}\left(g_{\alpha \beta}, \partial_{\varepsilon} g_{\alpha \beta}, \ldots, \partial_{\varepsilon_{1}} \ldots \partial_{\varepsilon_{n}} g_{\alpha \beta}, \ldots\right)$ which leads to second order field equations for minimally coupled sources is variationally equivalent to (44).

Equations (41), (44) enable us to decompose (31) as

where

$$
\boldsymbol{Q}_{\alpha}{ }^{\beta \gamma \delta}=\sqrt{-g} n Q_{\alpha}^{\beta \gamma \delta}-(16 \pi)^{-1} \sqrt{-g} g^{\beta[\gamma} \delta_{\alpha}^{\delta]},
$$

$$
Q_{\alpha}^{\beta \gamma \delta}=\partial L_{1} / \partial R_{\beta \gamma \delta}^{\alpha}
$$

is the quadrupole moment per particle [cf. (19)]. The first of (12) yields, in view of the homogeneity of $\boldsymbol{L}_{1}$,

$$
\left(\partial \boldsymbol{L} / \partial \boldsymbol{N}^{\alpha}\right) \boldsymbol{N}^{\alpha} \delta_{\varrho}^{\sigma}-\boldsymbol{t}_{\varrho}{ }^{\sigma}=\left(\partial \boldsymbol{L}_{1} / \partial \dot{e}_{\alpha}^{(a)}\right) e_{\alpha \mid \varrho}^{(a)} u^{\sigma}-\boldsymbol{L}_{3} \delta_{\varrho}^{\sigma}-\sqrt{-g} t_{\varrho}{ }^{\sigma},
$$

where

$$
\sqrt{-g} t_{\varrho}^{\sigma}=\boldsymbol{L}_{2} \delta_{\varrho}^{\sigma}-\phi_{A \mid \varrho} \partial \boldsymbol{L} / \partial \phi_{A \mid \sigma}
$$

gives the canonical energy tensor for the fields $\phi_{A}$. Since

$$
\partial \dot{e}_{\alpha}^{(a)} / \partial N^{\varrho}=e_{\alpha \mid \mu}^{(a)}\left(\partial u^{\mu} / \partial N^{\varrho}\right)=(\sqrt{-g} n)^{-1} e_{\alpha \mid \mu}^{(a)}\left(\delta_{\varrho}^{\mu}+u^{\mu} u_{\varrho}\right)
$$

we also have (noting that $\boldsymbol{L}_{1}$ is a function of $\dot{e}_{\alpha}^{(a)}$ whereas $\boldsymbol{L}$ was expressed as a function of $\left.e_{\alpha \mid \varrho}^{(a)}\right)$

$$
\left(\partial \boldsymbol{L} / \partial \boldsymbol{N}^{\varrho}\right) \boldsymbol{N}^{\sigma}=\left(\partial \boldsymbol{L}_{1} / \partial \dot{e}_{\alpha}^{(a)}\right) e_{\alpha \mid \varrho}^{(a)} u^{\sigma}+P_{\varrho} \boldsymbol{N}^{\sigma},
$$

where $P_{\varrho}$, defined by $(25)$, is the canonical momentum per particle.

Substituting (45), (47), and (49) into (40), we arrive at the final form of the gravitational field equations for a dustlike material:

$$
\begin{gathered}
-(8 \pi)^{-1} G_{\varrho}^{\sigma}=T_{\varrho}^{\sigma} \equiv P_{\varrho} n u^{\sigma}+t_{\varrho}{ }^{\sigma}+\frac{1}{2}(-g)^{-\frac{1}{2}}\left(\boldsymbol{S}^{\sigma \tau}{ }_{\varrho}+S_{\varrho}{ }^{\tau \sigma}+S^{\sigma}{ }_{\varrho}{ }^{\tau}\right)_{\mid \tau} \\
\\
+4\left(n Q^{\lambda}{ }_{(\varrho) \mu}{ }^{\sigma) \mu}\right)_{\mid \mu \lambda}+n Q^{A}\left(I_{A}{ }^{B}\right)_{\varrho}{ }^{\sigma} R_{B} .
\end{gathered}
$$

Here, $G_{Q}^{\sigma}$ is the Einstein tensor, and the last term is interpreted according to (39). Equation (50) identifies $T^{\varrho \sigma}$ as the "correct" (symmetric, covariantly constant) total energy-momentum tensor for material and fields. By virtue of (34) and (12), the spin flux can be decomposed into matter and field parts:

$$
\begin{gathered}
(-g)^{-\frac{1}{2}} \boldsymbol{S}^{\sigma \varrho \tau}=n S^{\sigma \varrho} u^{\tau}+S^{\sigma \varrho \tau}, \\
\frac{1}{2} \sqrt{-g} S_{\varrho}^{\sigma}{ }_{\varrho}^{\tau} \equiv\left(\partial \boldsymbol{L} / \partial \phi_{A \mid \tau}\right)\left(I_{A}{ }^{B}\right)_{[\varrho}{ }^{\sigma]} \phi_{B},
\end{gathered}
$$

with the spin angular momentum $S^{\sigma \varrho}$ per particle defined by $(25)$.

Recalling the definitions (10) and (20), we obtain from (41) and (43) the relation

$$
\frac{\delta \boldsymbol{L}}{\delta e_{\sigma}^{(a)}}=\sqrt{-g} n\left(\frac{\partial L_{1}}{\partial e_{\sigma}^{(a)}}+e_{(a)}^{\sigma} L_{1}\right)-\frac{\partial L_{1}}{\partial \dot{e}_{\sigma}^{(a)}} \boldsymbol{N}_{\mid \mu}^{\mu}+2 \frac{\partial\left(\boldsymbol{L}_{2}+\boldsymbol{L}_{3}\right)}{\partial g_{\varrho \sigma}} e_{(a) \varrho}
$$

between the variational derivatives of $\boldsymbol{L}$ and $L_{1}$. To the assumptions (i) and (ii) already made in this section we now add a final assumption:

(iii) The number of particles is conserved:

$$
N_{\mid \mu}^{\mu}=0 \text {. }
$$


Then (53) shows that the spin Eq. (36) is equivalent to the single-particle Eq. (20) and we thus recover the spin angular momentum equations of motion in the form (22). In the present context they specialize to

where

$$
\begin{aligned}
\frac{1}{2} \delta S^{\varrho \sigma} / \delta \tau=P^{[\varrho} u^{\sigma]} & +\left(I_{A}{ }^{B}\right)^{[\varrho \sigma]}\left(M^{A} \phi_{B}+M^{A \alpha} \phi_{B \mid \alpha}\right) \\
& -M^{A[\sigma} \phi_{A}{ }^{l \varrho]}+4 R^{[\sigma}{ }_{\alpha \beta \gamma} Q^{\varrho] \alpha \beta \gamma},
\end{aligned}
$$

$$
M^{A}=\partial L_{1} / \partial \phi_{A}, \quad M^{A \sigma}=\partial L_{1} / \partial \phi_{A \mid \sigma}
$$

and we have made use of (4a) and (39).

\section{Equations of Translational Motion Derived from "Variation of World-Lines"}

The four equations of motion for the canonical momentum $P_{\alpha}$ are implicitly contained in the preceding equations; they can be extracted by applying the contracted Bianchi identity $G_{\varrho \mid \sigma}^{\sigma}=0$ to (50) and simplifying with the aid of the spin Eq. (55) and the field Eq. (38). A simpler approach, which at the same time gives a variational characterization of these equations, is to "vary world-lines" 8 in the action integral (27).

The equations

$$
\partial x^{\mu}\left(a^{m}, t\right) / \partial t=v^{\mu}=u^{\mu} d \tau / d t
$$

define the world-lines as integral curves of the conserved particle flux $\boldsymbol{N}^{\mu}$. The three $a^{m}$ are "comoving" parameters $\left(u^{\mu} \partial_{\mu} a^{m}=0\right)$ and $t(\tau)$ is an arbitrary parameter along the curves.

The number of particles in a given infinitesimal flux tube $d^{3} a$ is a constant of the motion, and is given by

$$
N\left(a^{m}\right) d^{3} a=\left(-u^{\mu} n_{\mu}\right) n d \Sigma,
$$

where $d \Sigma$ is the 3 -area of a tube section $t=$ const and $n_{\mu}$ its unit normal. Since

$$
\sqrt{-g} d^{4} x=\left(-v^{\mu} n_{\mu}\right) d \Sigma d t
$$

we have

$$
N^{\mu} d^{4} x=N v^{\mu} d^{3} a d t
$$

from which

$$
\boldsymbol{L}_{1}\left(\boldsymbol{N}^{\mu}, \ldots\right) d^{4} x=N d^{3} a L_{1}\left(v^{\mu}, \ldots\right) d t
$$

follows by homogeneity of $\boldsymbol{L}_{1}$.

Consider now a 1-parameter family of flux and tetrad fields $N^{\mu}(x, \varepsilon), e_{\alpha}^{(a)}(x, \varepsilon)$ and let $x^{\mu}\left(a^{m}, t, \varepsilon\right)$ be the associated congruences. We extremize the action integral $I(\varepsilon)$ of (27) subject to fixed end-points $x^{\mu}\left(a^{m}, t_{i}, \varepsilon\right)=x^{\mu}\left(a^{m}, t_{i}, 0\right)(i=1,2)$ and to $\delta e_{\alpha}^{(a)} / \delta \varepsilon=0, \partial N / \partial \varepsilon=0$. The last equation constrains the number of particles in a flux tube to remain fixed when the tube is "varied". Under these conditions

$$
d I / d \varepsilon=\int d^{3} a N\left(a^{m}\right) \int\left\{d L_{1}\left(v^{\mu}, \ldots\right) / d \varepsilon\right\} d t
$$

8 For the application of this technique to non-spinning material, cf. Weyl [34], Taub [24], and Hawking and Ellis [24]. 
and a short calculation patterned after that of Section III gives the translational equations of motion (23) in the particular form

$$
\begin{aligned}
\delta P_{\mu} / \delta \tau= & \frac{1}{2} R_{\varrho \sigma \lambda \mu} S^{\varrho \sigma} u^{\lambda}+M^{A} \phi_{A \mid \mu}+M^{A \lambda} \phi_{A \mid \lambda \mu} \\
& +Q_{\alpha}{ }^{\beta \gamma \delta} R_{\beta \gamma \delta \mid \mu}^{\alpha} .
\end{aligned}
$$

The stress-free character of the medium is reflected in the absence of contributions to the variation (59) arising from the spatial dilation and deformation of the displaced world-tubes.

\section{Example: Einstein-Lorentz Theory for Spinning Charges and Dielectrics}

As an illustration of the results of Section IV, we here consider a charged dipolar medium and its interaction with a Maxwell-Einstein field, described by a vector potential $\phi_{\alpha}$ and the metric tensor.

The phenomenological (convective) current ${ }^{9} j^{\alpha}$, the polarization per particle $M^{\alpha \beta}$ and the "displacement tensor" $H^{\alpha \beta}$ (both skew-symmetric) are defined by the equations

$$
\begin{gathered}
\sqrt{-g} j^{\alpha}=\partial \boldsymbol{L} / \partial \phi_{\alpha}, \quad \sqrt{-g} n M^{\alpha \beta}=\partial \boldsymbol{L}_{1} / \partial \phi_{\beta \mid \alpha}=2 \partial \boldsymbol{L}_{1} / \partial F_{\alpha \beta}, \\
\sqrt{-g} H^{\alpha \beta}=-8 \pi \partial \boldsymbol{L} / \partial F_{\alpha \beta},
\end{gathered}
$$

in which it has been assumed that $\phi_{\alpha \mid \beta}$ enters the Lagrangian only through the skew-symmetric combination $F_{\alpha \beta}=2 \partial_{[\alpha} \phi_{\beta]}$. The electromagnetic field equations are therefore [cf. (38)]

$$
\partial_{[\alpha} F_{\beta \gamma]}=0, \quad H_{\mid \beta}^{\alpha \beta}=4 \pi j^{\alpha}
$$

and imply conservation of free charge

$$
j^{\alpha}{ }_{\alpha}=0 \text {. }
$$

From (48) and (52) we obtain

$$
t_{\varrho}{ }^{\sigma}=(-g)^{-\frac{1}{2}} \boldsymbol{L}_{2} \delta_{\varrho}^{\sigma}-(4 \pi)^{-1} \phi_{\alpha \mid \varrho} H^{\alpha \sigma}, 4 \pi S^{\varrho \sigma \tau}=2 \phi^{[\varrho} H^{\sigma] \tau}
$$

so that

$$
t_{\varrho}{ }^{\sigma}+\frac{1}{2}\left(S^{\sigma \tau}{ }_{\varrho}+S_{\varrho}^{\tau \sigma}+S_{\varrho}^{\sigma}{ }_{\varrho}^{\tau}\right)_{\mid \tau}=T_{\varrho}^{(\mathrm{e} . \mathrm{m} .) \sigma}-\phi_{\varrho} j^{\sigma},
$$

where we have defined a gauge-invariant electromagnetic energy tensor by (cf. $[1,22,25]$ )

$$
T_{\varrho}^{(\mathrm{e} . \mathrm{m} .) \sigma}=(4 \pi)^{-1} F_{\varrho \alpha} H^{\sigma \alpha}+(-g)^{-\frac{1}{2}} \boldsymbol{L}_{2} \delta_{\varrho}^{\sigma} .
$$

We now assume more specifically:

(i) that the free-field electromagnetic Lagrangian is

$$
\boldsymbol{L}_{2}=-(16 \pi)^{-1} \sqrt{-g} F_{\mu \nu} F^{\mu \nu},
$$

which leads at once to the usual Lorentz polarization relations

$$
H^{\alpha \beta}=F^{\alpha \beta}-4 \pi n M^{\alpha \beta}
$$

9 The "true" (microscopic) current is $J^{\alpha} \equiv(-g)^{-\frac{1}{2}} \delta \boldsymbol{L}_{1} / \delta \phi_{\alpha}=j^{\alpha}\left(n M^{\alpha \beta}\right)_{\mid \beta}$. 
and (ii) that the (undifferentiated) potential $\phi_{\alpha}$ enters $\boldsymbol{L}$ only through a bilinear interaction term $e \phi_{\alpha} N^{\alpha}$, whence

$$
j^{\alpha}=e n u^{\alpha}
$$

and $d e / d \tau=0$ as a consequence of (54) and (63). In terms of a gauge-invariant "kinetic momentum"

$$
p_{\alpha}=P_{\alpha}-e \phi_{\alpha}
$$

the equations of motion (55), (60) reduce to ${ }^{10}$ (cf. $\left.[8,9,14,15]\right)$

$$
\begin{gathered}
\delta p_{\mu} / \delta \tau=-\frac{1}{2} R_{\mu \alpha \beta \gamma} u^{\alpha} S^{\beta \gamma}+F_{\mu \alpha} e u^{\alpha}+\frac{1}{2} M^{\alpha \beta} F_{\alpha \beta \mid \mu}+Q_{\alpha}^{\beta \gamma \delta} R_{\beta \gamma \delta \mid \mu}^{\alpha}, \\
\frac{1}{2} \delta S^{\lambda \mu} / \delta \tau=p^{[\lambda} u^{\mu]}-F_{\alpha}^{[\lambda} M^{\mu] \alpha}-4 R_{\alpha \beta \gamma}^{[\lambda} Q^{\mu] \alpha \beta \gamma} .
\end{gathered}
$$

Insertion of (65) and (51) into the gravitational field Eq. (50) gives

$$
\begin{aligned}
-(8 \pi)^{-1} G_{\varrho}^{\sigma}=T_{\varrho}^{\sigma} & \equiv T_{\varrho}^{(\mathrm{e} . \mathrm{m} .) \sigma}+T_{\varrho}^{(\mathrm{mat}) \sigma}+\frac{1}{2}\left(S_{(\mathrm{mat}) \varrho}^{\sigma \tau}-S_{(\mathrm{mat}) \varrho}^{\tau}+S_{(\mathrm{mat}) \varrho}^{\sigma}\right)_{\mid \tau} \\
& +4\left(n Q_{(\varrho}^{\lambda}{ }^{\sigma) \mu}\right)_{\mid \mu \lambda}+n Q^{A}\left(I_{A}{ }^{B}\right)_{\varrho}{ }^{\sigma} R_{B}
\end{aligned}
$$

in which the tensors

$$
T_{\varrho}^{\text {(mat) } \sigma}=n p_{\varrho} u^{\sigma}, \quad S_{(\text {mat })}^{\varrho \sigma \tau}=n S^{\varrho \sigma} u^{\tau}
$$

represent the material fluxes of linear and spin angular momentum. Their divergences are, according to (72), (71), given by

$$
\begin{aligned}
& \frac{1}{2} S_{(\text {mat }) \mid \tau}^{\varrho \sigma \tau}=T_{(\text {mat) }}^{[\varrho \sigma]}-F^{\left[\varrho_{\alpha}\right.} M^{\sigma] \alpha}-4 R^{[\varrho}{ }_{\alpha \beta \gamma} n Q^{\sigma] \alpha \beta \gamma}, \\
& \left(T^{\text {(mat) }}+T^{(\mathrm{e} . \mathrm{m} .)}\right)_{\varrho \mid \sigma}{ }^{\sigma}=-\frac{1}{2} R_{\varrho \alpha \beta \gamma} S_{(\mathrm{mat})}^{\beta \gamma \alpha}+n Q_{\alpha}{ }^{\beta \gamma \delta} R_{\beta \gamma \delta \mid \varrho}^{\alpha},
\end{aligned}
$$

where we have used the result

$$
T_{\varrho}^{(\mathrm{e} . \mathrm{m} .) \sigma}{ }_{\mid \sigma}=-F_{\varrho \alpha} j^{\alpha}-\frac{1}{2} n M^{\mu v} F_{\mu \nu \mid \varrho}
$$

which follows from (66), (68), and (62). These equations can be used to verify directly that the total energy tensor $T_{\varrho}^{\sigma}$ is symmetric and conserved, as required for consistency of the gravitational field equations.

The electromagnetic and gravitational field equations, here derived for a dustlike medium, should retain their form in more general circumstances [with appropriate generalization of the expressions (74) for the material terms]. This question will not be pursued further here (but compare Refs. [1, 18, 19, 22, 25]).

We conclude this section with some brief comments on the equations of motion (71),(72). For determinacy these have to be supplemented by three equations for the normalized 4-velocity $u^{\mu}$. The form of these equations will depend on the detailed functional form of the Lagrangian and constraints (if any) imposed on the

10 These equations become those of Refs. [1] and [2] if one sets $Q_{\alpha}{ }^{\beta \gamma \delta}=0$ and redefines the kinetic momentum as $p_{\alpha}^{(\text {new })}=p_{\alpha}+F_{\alpha \beta} q^{\beta}$, where $q^{\beta}$ is the electric dipole moment. This merely amounts to a different convention for allocating the interaction energy between matter and field, and leads to field equations equivalent to (62) and (73). The present formulation, which, in effect, is that of Ref. [1] with $q^{\beta}$ set equal to zero, appears to be the simplest and most natural from a formal point of view. 
variation. (In the multipole analysis of extended bodies $([2,8-10])$ the equations for $u^{\mu}$ amount to a convention for fixing the centre-of-mass.) In this paragraph we shall follow current practice $([2,8,9,14,15])$ and adopt as the supplementary conditions

$$
S^{\lambda \mu} p_{\mu}=0
$$

The proper-time derivative of (78), written out with the aid of (71) (abbreviated for convenience to $\dot{p}_{\mu}=F_{\mu}$ ) and (72), yields three algebraic equations for $u^{\mu}$ in terms of $p_{\mu}, S^{\lambda \mu}$ and the applied fields. These equations take the form

$$
F_{\mu} S^{\mu \lambda}=p_{\mu}\left(p^{\lambda} u^{\mu}-p^{\mu} u^{\lambda}\right)+\kappa(\tau) p_{\mu} F^{\mu \alpha} S_{\alpha}^{\lambda}
$$

for the special case of magnetic-dipole gravitational-monopole charges with gyromagnetic ratio proportional to $\kappa(\tau)$ :

$$
Q_{\alpha}^{\beta \gamma \delta}=0, \quad M^{\alpha \beta}=\kappa(\tau) S^{\alpha \beta} .
$$

From (72) and (80) we obtain at once the conservation of spin energy

$$
(d / d \tau)\left(S_{\lambda \mu} S^{\lambda \mu}\right)=0 .
$$

Transvection of (79) with $F_{\lambda}$, a second use of (79) and of the equation

$$
F_{\mu} u^{\mu}=\frac{1}{2} \kappa(\tau) S^{\alpha \beta} F_{\alpha \beta \mid \mu} u^{\mu}
$$

following from (71), yields

$$
F_{\lambda} p^{\lambda}=\frac{1}{2} \kappa(\tau)\left(p_{\lambda} p^{\lambda} / p_{\mu} u^{\mu}\right)(d / d \tau)\left(S^{\alpha \beta} F_{\alpha \beta}\right)
$$

From this we can infer a conservation law

$$
(d / d \tau)\left(-p_{\lambda} p^{\lambda}+g S^{\alpha \beta} F_{\alpha \beta}\right)=0
$$

if we assume that $\kappa(\tau)=g\left(p_{\mu} u^{\mu} / p_{\lambda} p^{\lambda}\right)$ and $g=$ constant. Comparison with the nonrelativistic limit indicates that (82) should be interpreted as expressing conservation of proper mass.

\section{Field Dynamics of Continua: Higher-derivative Coupling}

We shall now outline briefly how the considerations of Section IV can be extended to the case where the Lagrangian includes higher derivatives of the fields.

It will be convenient to use the following notation: a bold face index $\alpha(n)$ denotes the symmetrized set of indices $\left(\alpha_{1} \ldots \alpha_{n}\right) ; \alpha(0)$ denotes the empty set. Repeated bold face indices imply summation. Thus,

$$
X^{A \boldsymbol{\alpha}(n)} Y_{B \boldsymbol{\alpha}(n)}=\left\{\begin{array}{lll}
X^{A} Y_{B} & \text { if } & n=0 \\
X^{A\left(\alpha_{1} \ldots \alpha_{n}\right)} Y_{B\left(\alpha_{1} \ldots \alpha_{n}\right)} & \text { if } & n=1,2, \ldots
\end{array}\right.
$$




\section{Generalization of Fundamental Identity (14)}

We consider an arbitrary scalar density $\boldsymbol{L}\left(\Psi_{\boldsymbol{A}}\right)$ where $\Psi_{\boldsymbol{A}}=\left\{\psi_{\boldsymbol{A} \mid \boldsymbol{x}(n)}, n=0,1, \ldots\right\}$, and extend definitions (11), (12) as follows:

$$
\begin{gathered}
\boldsymbol{L}^{A} \equiv \partial \boldsymbol{L} / \partial \psi_{A}, \boldsymbol{L}^{A \alpha_{1} \ldots \alpha_{n}} \equiv \partial \boldsymbol{L} / \partial \psi_{A \mid\left(\alpha_{1} \ldots \alpha_{n}\right)}=\boldsymbol{L}^{A \boldsymbol{\alpha}(n)}, \\
\boldsymbol{L}_{*}^{A \alpha_{1} \ldots \alpha_{n}} \equiv \sum_{m=0}^{\infty}(-1)^{m} \boldsymbol{L}_{\mid \boldsymbol{\beta}(m)}^{A \boldsymbol{\alpha}(n) \boldsymbol{\beta}(m)}=\boldsymbol{L}_{*}^{A \boldsymbol{\alpha}(n)}, \\
\boldsymbol{t}_{\varrho}{ }^{\sigma} \equiv \delta_{\varrho}^{\sigma} \boldsymbol{L}-\sum_{n=0}^{\infty}(n+1) \psi_{A \mid(\varrho \boldsymbol{\alpha}(n))} \boldsymbol{L}^{A \sigma \boldsymbol{\alpha}(n)}, \\
\boldsymbol{U}_{\varrho}^{\tau \sigma}{ }_{\varrho} \equiv\left(I_{A}{ }^{B}\right)_{\varrho}{ }^{\sigma} \sum_{n=0}^{\infty} \boldsymbol{L}_{*}^{A \tau \boldsymbol{\alpha}(n)} \psi_{B \mid \boldsymbol{\alpha}(n)} .
\end{gathered}
$$

From (84) it follows at once that

$$
\boldsymbol{L}_{*}^{A \tau \boldsymbol{\alpha}(n)}{ }_{\mid \tau}=\boldsymbol{L}^{A \boldsymbol{\alpha}(n)}-\boldsymbol{L}_{*}^{A \boldsymbol{\alpha}(n)} .
$$

Hence the divergence of (86) yields

$$
\boldsymbol{U}^{\tau \sigma}{ }_{\varrho \mid \tau}+\boldsymbol{L}_{*}^{A}\left(I_{A}{ }^{B}\right)_{\varrho}{ }^{\sigma} \psi_{B}=\left(I_{A}{ }^{B}\right)_{\varrho}{ }^{\sigma} \sum_{n=0}^{\infty} \boldsymbol{L}^{A \boldsymbol{\alpha}(n)} \psi_{B \mid \boldsymbol{\alpha}(n)} .
$$

On the other hand, repeated application of (4a) leads to

$$
\begin{gathered}
\boldsymbol{L}^{A \boldsymbol{\alpha}(n)}\left(I_{A \boldsymbol{\alpha}(n)}{ }^{B \boldsymbol{\beta}(n)}\right)_{\varrho}{ }^{\sigma} \psi_{B \mid \boldsymbol{\beta}(n)}=\boldsymbol{L}^{A \boldsymbol{\alpha}(n)}\left(I_{A}{ }^{B}\right)_{\varrho}{ }^{\sigma} \psi_{B \mid \boldsymbol{\alpha}(n)} \\
-n \boldsymbol{L}^{A \sigma \boldsymbol{\alpha}(n-1)} \psi_{A \mid(\varrho \boldsymbol{\alpha}(n-1))}, \quad(n=1,2, \ldots)
\end{gathered}
$$

which enables us to write the general identity (9) in the form

$$
\boldsymbol{t}_{\varrho}{ }^{\sigma}=-\left(I_{A}{ }^{B}\right)_{\varrho}{ }^{\sigma} \sum_{n=0}^{\infty} \boldsymbol{L}^{A \boldsymbol{\alpha}(n)} \psi_{B \mid \boldsymbol{\alpha}(n)},
$$

where use has been made of (85).

From (88) and (90), we obtain the generalized form of identity (14),

$$
\boldsymbol{U}^{\tau \sigma}{ }_{\varrho \mid \tau}+\boldsymbol{L}_{*}^{A}\left(I_{A}{ }^{B}\right)_{\varrho}{ }^{\sigma} \psi_{B}+\boldsymbol{t}_{\varrho}{ }^{\sigma}=0,
$$

applicable to a scalar density $\boldsymbol{L}\left(\psi_{A \mid \boldsymbol{\alpha}(n)}\right)$ depending on (symmetrized) covariant derivatives of arbitrary order.

\section{Generalized Action Integral; Variation of Tetrad}

As a generalization of the action integral (27), we now consider

$$
I=\int \boldsymbol{L}\left(\psi_{A \mid \boldsymbol{\alpha}(n)}\right) d^{4} x, \psi_{A} \equiv\left\{\boldsymbol{N}^{\alpha}, e_{\alpha}^{(a)}, \phi_{A}, R_{\beta \gamma \delta}^{\alpha}\right\},
$$

in which arbitrary symmetrized derivatives of the field variables $\phi_{A}, R_{\beta \gamma \delta}^{\alpha}$ are now permitted to appear, but we still assume that $\boldsymbol{N}^{\alpha}$ occurs only in undifferentiated form, and second and higher derivatives of $e_{\alpha}^{(a)}$ are absent. 
Under a variation of the tetrad field, we have

$$
\delta_{(e)} \boldsymbol{L}=\sum_{n=0}^{\infty} \boldsymbol{L}^{A \boldsymbol{\alpha}(n)} \delta_{(e)}\left(\psi_{A \mid \boldsymbol{\alpha}(n)}\right) .
$$

We now observe from (6) that

$$
\begin{gathered}
\boldsymbol{L}^{A \boldsymbol{\alpha}(n)} \delta_{(e)}\left(\psi_{A \mid \boldsymbol{\alpha}(n)}\right)=\boldsymbol{L}^{A \boldsymbol{\alpha}(n)}\left[\left\{\delta_{e}\left(\psi_{A \mid \boldsymbol{\alpha}(n-1)}\right)\right\}_{\mid \alpha_{n}}\right. \\
\left.\quad+\left(I_{A \boldsymbol{\alpha}(n-1)}{ }^{B \boldsymbol{\beta}(n-1)}\right)_{\varrho}{ }^{\sigma} \psi_{B \mid \boldsymbol{\beta}(n-1)} \delta_{(e)} \Gamma_{\sigma \alpha_{n}}^{\varrho}\right],
\end{gathered}
$$

make use of (89) and carry out $n$ "integrations by parts". Then (93) becomes

$$
\delta_{(e)} \boldsymbol{L}=\boldsymbol{L}_{*}^{A} \delta_{(e)} \psi_{A}+\boldsymbol{U}_{* \varrho}^{\tau \sigma} \delta_{(e)} \Gamma_{\sigma \tau}^{\varrho}+(\text { div })
$$

where we have defined

$$
\boldsymbol{U}_{* \varrho}^{\tau \sigma} \equiv \boldsymbol{U}_{\varrho}^{\tau \sigma}-\boldsymbol{Y}_{\varrho}^{\tau \sigma} ; \boldsymbol{Y}^{\tau \sigma}{ }_{\varrho} \equiv \sum_{n=0}^{\infty}(n+1) \psi_{A \mid(\varrho \boldsymbol{\alpha}(n))} \boldsymbol{L}_{*}^{A \sigma \tau \boldsymbol{\alpha}(n)} .
$$

According to the general identity (32), we have

$$
\boldsymbol{L}_{*}^{A} \delta_{(e)} \psi_{A}=\left(\delta \boldsymbol{L} / \delta e_{\sigma}^{(a)}\right) \delta e_{\sigma}^{(a)}-2 \boldsymbol{Q}_{\varrho * \mid \mu}^{\sigma \mu \tau} \delta_{(e)} \Gamma_{\sigma \tau}^{\varrho}+(\mathrm{div}),
$$

in which $Q_{\varrho *}^{\sigma \mu \tau}$ is derived from $Q_{\varrho}{ }^{\sigma \mu \tau}$ by the prescription (84).

The action principle $\delta_{(e)} I=0$ thus leads, with the aid of the general identity (33), to the generalization of (35):

$$
\left(\delta \boldsymbol{L} / \delta e_{\sigma}^{(a)}\right) e^{(a) \varrho}=\left\{\boldsymbol{U}_{*}^{\tau(\varrho \sigma)}-\frac{1}{2}\left(\boldsymbol{S}_{*}^{\sigma \tau \varrho}+\boldsymbol{S}_{*}^{\varrho \tau \sigma}\right)\right\}_{\mid \tau}-4 \boldsymbol{Q}_{*}^{\lambda(\varrho \sigma) \mu}{ }_{\mid \mu \lambda},
$$

where

$$
S_{*}^{\rho \sigma \tau} \equiv 2 U_{*}^{\tau[\rho \sigma]}
$$

We thus recover the spin equations in the form (36).

\section{The Field Equations}

If we now eliminate $\left(\delta \boldsymbol{L} / \delta e_{\sigma}^{(a)}\right) e_{\varrho}^{(a)}$ between (96) and (91), we arrive at the gravitational field equations in the form

$$
\begin{gathered}
\left(\partial \boldsymbol{L} / \partial \boldsymbol{N}^{\alpha}\right) \boldsymbol{N}^{\alpha} \delta_{\varrho}^{\sigma}-\boldsymbol{t}_{\varrho}{ }^{\sigma}-\boldsymbol{Q}_{*}^{A}\left(I_{A}{ }^{B}\right)_{\varrho}{ }^{\sigma} R_{B}=\left(\partial \boldsymbol{L} / \partial \boldsymbol{N}^{\varrho}\right) \boldsymbol{N}^{\sigma} \\
\quad+\frac{1}{2}\left(\boldsymbol{S}_{* \varrho}^{\sigma \tau}+\boldsymbol{S}_{\varrho *}^{\tau \sigma}+\boldsymbol{S}_{* \varrho}^{\sigma}{ }^{\tau}\right)_{\mid \tau}+4 \boldsymbol{Q}_{*}^{\lambda\left(\sigma{ }^{\prime}{ }^{\mu} \mid \mu \lambda\right.}+\boldsymbol{Y}^{\tau \sigma}{ }_{\varrho \mid \tau}
\end{gathered}
$$

modulo the non-gravitational field equations

$$
\delta \boldsymbol{L} / \delta \phi_{A} \equiv \boldsymbol{L}_{*}^{A}(\phi)=0 .
$$

To throw these into a more familiar form, we follow the pattern of Section IV, 2 : we decompose $\boldsymbol{L}$ as

$$
\begin{aligned}
\boldsymbol{L}= & n \sqrt{-g} L_{1}\left(u^{\mu}, e_{\alpha}^{(a)}, \dot{e}_{\alpha}^{(a)}, \phi_{A \mid \boldsymbol{\alpha}(n)}, R_{A \mid \boldsymbol{\alpha}(n)}\right) \\
& +\boldsymbol{L}_{2}\left(\phi_{A \mid \boldsymbol{\alpha}(n)}, g_{\alpha \beta}\right)-(16 \pi)^{-1} \sqrt{-g} R
\end{aligned}
$$


where $L_{1}$ is assumed homogeneous of first degree in its first argument. Equation (98) is then found to reduce to

$$
\begin{aligned}
-(8 \pi)^{-1} G_{\varrho}^{\sigma}= & T_{\varrho}^{\sigma} \equiv P_{\varrho} n u^{\sigma}+t_{\varrho}{ }^{\sigma}+\frac{1}{2}(-g)^{-\frac{1}{2}}\left(S_{* \varrho}^{\sigma \tau}+S_{\varrho *}^{\tau \sigma}+S_{* \varrho}^{\sigma}{ }^{\tau}\right)_{\mid \tau} \\
& +(-g)^{-\frac{1}{2}}\left(4 Q_{*(\varrho}{ }^{\lambda}{ }^{\sigma) \mu} \mid \mu \lambda\right. \\
& +Q_{*}^{A}\left(I_{A}{ }^{B}\right)_{\varrho}{ }^{\sigma} R_{B} \\
& \left.-\sum_{m=0}^{\infty} Q_{*}^{A \sigma \alpha(m)} R_{A \mid(\varrho \alpha(m))}\right) .
\end{aligned}
$$

In this equation, the canonical momentum per particle, $P_{\mu}$, is given by $(25)$; the gravitational multipole moments per particle $Q^{A \alpha_{1} \ldots \alpha_{n}}$ and the associated quantities $\boldsymbol{Q}_{*}^{A}$ are defined by

$$
\begin{gathered}
n^{-1}(-g)^{-\frac{1}{2}} \boldsymbol{Q}^{A \alpha_{1} \ldots \alpha_{n}}=Q^{A \alpha_{1} \ldots \alpha_{n}}=\partial L_{1} / \partial R_{A \mid \boldsymbol{\alpha}(n)}, \\
\boldsymbol{Q}_{*}^{A \boldsymbol{\alpha}(n)}=\sum_{m=0}^{\infty}(-1)^{m} \boldsymbol{Q}^{A \boldsymbol{\alpha}(n) \boldsymbol{\beta}(m)}{ }_{\mid \boldsymbol{\beta}(m)},
\end{gathered}
$$

and

$$
\sqrt{-g} t_{\varrho}^{\sigma}=\boldsymbol{L}_{2} \delta_{\varrho}^{\sigma}-\sum_{m=0}^{\infty} \phi_{A \mid(\varrho \alpha(m))} \boldsymbol{L}_{*}^{A \sigma \alpha(m)}(\phi)
$$

(where $\left.\boldsymbol{L}_{*}^{A \sigma \alpha(m)}(\phi) \equiv \sum_{n=0}^{\infty}(-1)^{n}\left(\partial \boldsymbol{L} / \partial \phi_{A \mid(\sigma \alpha(m) \boldsymbol{\beta}(n))}\right)_{\mid \boldsymbol{\beta}(n)}\right)$ gives the canonical energy tensor for the fields $\phi_{A}$ [35]. As in (51), the total spin flux $S_{*}^{\sigma \varrho \tau}$ can be split into a matter part $n S^{\varrho \sigma} u^{\tau}$, and a field part obtainable from (97), (95), and (86) which is now more complicated than (52) and includes contributions from the curvature.

Acknowledgements. We are indebted to Karel Kuchar and Hans Kunzle for helpful discussions.

\section{References}

1. Israel, W.: Lettere al Nuovo Cimento 7, 860 (1973)

2. Suttorp, L.G., de Groot, S. R.: Foundations of electrodynamics, Chapters 4, 5, § 7, Amsterdam: North-Holland 1972

3. Halbwachs, F.: Théorie relativiste des fluides a spin. Paris: Gauthier-Villars 1960

4. Corben, H.C.: Classical and quantum theories of spinning particles. New York: Holden-Day 1967

5. Mathisson, M.: (a) Z. Physik 67, 826 (1931); (b) Proc. Cambridge Phil. Soc. 38, 40 (1942)

6. Papapetrou, A.: (a) Praktika de l'Akademie d'Athenes 14, 540 (1939); (b) Proc. Roy. Soc. London A209, 248 (1951)

7. Taub, A.H.: (a) J. Math. Phys. 5, 112 (1964); (b) Proceedings of the Galileo Galilei centenary meeting on general relativity, p. 1. Firenze: G. Barbera 1965

8. Dixon, W. G.: (a) Nuovo Cimento 34, 317 (1964); (b) J. Math. Phys. 8, 1591 (1967); (c) Proc. Roy. Soc. London A314, 499 (1970); A319, 509 (1970)

9. Madore, J. : (a) Ann. Inst. Henri Poincaré 11, 221 (1969); (b) C. R. Acad. Sci. Paris 273A, 782 (1971)

10. Das, A.: Progr. Theoret. Phys. 23, 610 (1960); Tulczyjew, W.: Acta Phys. Polon. 18, 393 (1959); Westfahl, K.: Ann. Physik 20, 241 (1967)

11. Pryce, M.H.L.: Proc. Roy. Soc. London A195, 62 (1948); Møller, C.: Ann. Inst. Henri Poincaré 11, 251 (1949); Beiglböck, W.D.: Commun. math. Phys. 5, 106 (1967)

12. Frenkel, J.: Z. Physik 37, 243 (1926)

13. Barut, A.O.: Electrodynamics and classical theory of fields and particles, p. 77. New York: MacMillan 1964 
14. Künzle,H.P.: (a) J. Math. Phys. 13, 739 (1972); (b) Commun. math. Phys. 27, 23 (1972)

15. Duval, C., Fliche,H.H., Souriau, J. M.: C. R. Acad. Sci. Paris 274, 1082 (1972)

16. Bargmann, V., Michel, L., Telegdi, V.L.: Phys. Rev. Letters 2, 435 (1959); Weyssenhoff, J., Raabe, A.: Acta Phys. Polon. 9, 46 (1947)

17. Pauli, W.: Theory of relativity (transl. by G. Field, $\S 35$ and p. 216. New York: Pergamon 1958

18. Møller, C.: Theory of relativity, Chapter 7. Oxford: Clarendon Press 1972

19. Penfield, P., Haus, H. A.: Electrodynamics of moving media. Cambridge, Mass.: M. I. T. Press 1967

20. Minkowski, H.: (a) Nachr. Ges. Wiss. Göttingen 53 (1908); (b) Math. Ann. 68, 472 (1910); Abraham, M.: Ann. Physik 44, 537 (1914); Einstein, A., Laub, J.: Ann. Physik 26, 541 (1908)

21. de Groot, S.R., Suttorp, L. G.: Physica 39, 84 (1968)

22. Grot, R. A., Eringen, A.C.: Int. J. Engng. Sci. 4, 610 (1966)

23. Rosenfeld, L.: Mem. Acad. Roy. Belg. 18, fasc. 6 (1940); Belinfante, F. J.: Physica 6, 887 (1939)

24. Taub, A.H.: In: Cattaneo, C. (Ed.): Relativistic fluid dynamics, p. 266; Bressanone: C.I.M.E. 1971; Hawking, S.W., Ellis, G.F.R.: The large scale structure of space-time, p. 69. New York: Cambridge U.P. 1973

25. Maugin, G. A.: Ann. Inst. Henri Poincaré 15, 275 (1971)

26. Weyl,H.: Phys. Rev. 77, 699 (1950)

27. Sciama, D. W.: J. Math. Phys. 2, 472 (1961)

28. Kibble, T. W. B.: J. Math. Phys. 2, 212 (1961)

29. Hehl,F.W.: GRG Journ. 4, 333 (1973); 5, 491 (1974)

30. Trautman, A.: (a) Bull. Acad. Polon. Sci. 20, 185, 503 (1972); (b) Symposia Mathematica 12, 139 (1973)

31. Isham, C. J., Salam, A., Strathdee, J.: Nature (Phys. Sci.) 244, 82 (1973)

32. Trautman, A.: Nature (Phys. Sci.) 242, 7 (1973); Stewart, J., Hâjiček, P.: Nature (Phys. Sci.) 244, 96 (1973); Copzýnski, W.: Phys. Lett. A 43, 63 (1973)

33. Lovelock, D.: J. Math. Phys. 13, 874 (1972)

34. Weyl, H.: Space, time, matter, p. 230. New York: Dover 1951

35. De Wet, J. S.: Proc. Cambridge Phil. Soc. 44, 546 (1948); Chang, T. S.: Proc. Cambridge Phil. Soc. 44, 70 (1948)

Communicated by J. Ehlers

Ian Bailey

Werner Israel

Department of Physics

The University of Alberta

Edmonton T6G 2E1, Alberta, Canada 